\title{
An Adaptive Robust Control for Trajectory Tracking Of a Robotic Manipulator System
}

\author{
Bin Ren, Yao Wang, Lilan Liu, Xiaowei Tu, Rogelio Lozano
}

\begin{abstract}
The conventional adaptive control algorithm has a very high real-time requirement for six degrees-of-freedom (DOF) series robotic manipulator system. When the unknown parameters of the robotic manipulator are mutated, it is difficult to ensure the stability of the robotic manipulator system. Aiming at this phenomenon, an optimal algorithm based on adaptive robust control is proposed. When the algorithm is applied to the robotic manipulator system, the actual trajectory at the end of the manipulator is as close as possible to the desired trajectory in the simulation. The algorithm is based on the conventional algorithm, the design of the sliding mode surface to reduce the system position error, adding robust control algorithm to compensate for the instability of the system. The simulation results show that the actual trajectory can quickly track the desired trajectory, and the position error approaches zero.
\end{abstract}

Index Terms - six-DOF, adaptive control algorithm, robust control algorithm.

\section{INTRODUCTION}

The robotic manipulator is an inherently nonlinear, strongly coupled and time-varying system [1]. And the control design and stability analysis of robotic manipulator have received considerable attention. At the same time, there were many control strategies that have been proposed, such as the PID (proportional-integral-derivative) control, the sliding mode control, the adaptive control, the neural network control and so on. In fact, the robotic system is inherently nonlinear with partially known or unknown dynamics makes the use of conventional methods difficult [2]. There are numerous applications in industries like manufacturing, aerospace and medical in which they are used to pick up the appointed objects. Therefore, the accurate position tracking control is the core of the robotic system.

In [3], an improved adaptive robust control is proposed, which is based on the desired compensation adaptive robust control. This strategy uses the method of maximum likelihood parameter estimation to obtain the accurate results of parameter identification, and the integral parameter estimation is adjusted to the proportional integral parameter

Dr.Bin Ren, is currently an associate professor in Department of Mechanical Engineering, Shanghai Key Laboratory of Intelligent Manufacturing and Robotics, Shanghai University, China

Yao Wang, is with Department of Mechanical Engineering, Shanghai University, China

Xiaowei Tu and Lilan Liu, are with Shanghai University.

Rogelio Lozano, is with Universit de Technologie de Compiegne, CNRS UMR 7253 Heudiasyc, France.

Thanks for the support of the following funds: 1) The National Natural Science Foundation of China (No.51775325), 2) Yong Eastern Scholar (No. QD2016033), 3) Hong Kong Scholars Program of China (No.XJ2013015). estimation. The simulation results prove that the control has significantly improved the stability and controllability of this system. In [4], based on the conventional PID control, it adds an algorithm for estimating the equivalent rotational inertia. It proposes an APID (Adaptive PID) control algorithm. The simulation results prove that this algorithm has the advantages of stable tracking performance, high control precision and strong robustness compared to the conventional PID control. In [5], an adaptive neural synovial control is proposed in which it adds an adaptive control system for neural network error based on the traditional neural synovial control. The simulation results prove that this algorithm realize the automatic tracking of modeling error and uncertain interference, and improve the stability of the system. In [6], it combines the PD control with the feed forward control and there is an experiment act on a 2-DOF humanoid manipulator. The simulation results prove that this method successfully reduces the average absolute error of the robotic manipulator.

In this paper, we studied and learned lots of differently solutions and used the existing 6-DOF series robotic manipulator for research objects. We firstly analyzed the structure of the robotic manipulator, and used D-H (Denavit-Hartenberg) method to construct the transformation matrix between each joint of the robotic manipulator, and deduced the positive kinematics model of the robotic manipulator. Then, we optimized the adaptive control algorithm and used it for simulation and experiment. The simulation results proved that this algorithm improved the tracking performance of the system.

\section{KINEMATICS MODEL}

The motion analysis of the series robotic manipulator is to solve the position and posture of the hand claw knowing the motion parameters of each joint. In order to describe the movement of the robotic manipulator of the link, we usually use D-H method to establish the coordinate system [7]-[9].

In Fig.1, we set up the coordinate system of links $\{i\}$ ( $i=1,2 \ldots 6$ ), let ${ }_{i}^{i-1} T$ as the transformation of links of coordinate system $\{i\}$ relative to $\{i-1\}$. The transformation of links ${ }_{i}^{i-1} T$ can be regarded as the coordinate system $\{i\}$ is obtained though the following four transformation:

1) Revolved angle $\left(\theta_{i}\right)$ around the axis $\left(z_{i-1}\right)$;

2) Moved length $\left(d_{i}\right)$ along the axis $\left(z_{i-1}\right)$;

3) Moved length $\left(a_{i}\right)$ along the axis $\left(x_{i}\right)$;

4) Revolved angle $\left(\alpha_{i}\right)$ around the axis $\left(x_{i}\right)$. 


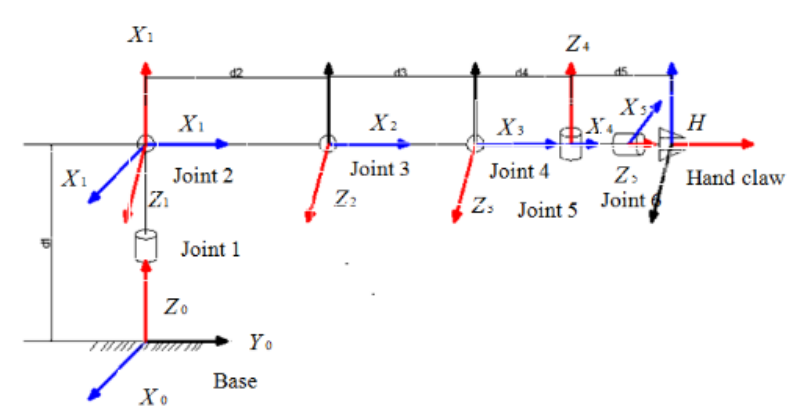

Fig.1. The coordinate system of links.

where $\theta$ represents the rotation angle around the $z$ axis, $d$ represents the distance between two adjacent common perpendicular lines on the $z$ axis, a represents the length of links, $\alpha_{i}$ represents the angle between two adjacent $z$ axis. Therefore,

$$
\begin{gathered}
{ }_{i}^{i-1} T=\operatorname{Rot}\left(z, \theta_{i}\right) \cdot \operatorname{Trans}\left(0,0, d_{i}\right) \cdot \\
\operatorname{Trans}\left(a_{i}, 0,0\right) \cdot \operatorname{Rot}\left(x, \alpha_{i}\right)
\end{gathered}
$$

where

$$
\begin{gathered}
\operatorname{Rot}\left(z, \theta_{i}\right)=\left[\begin{array}{cccc}
c \theta_{i} & -s \theta_{i} & 0 & 0 \\
s \theta_{i} & c \theta_{i} & 0 & 0 \\
0 & 0 & 1 & 0 \\
0 & 0 & 0 & 1
\end{array}\right] \\
\operatorname{Trans}\left(0,0, d_{i}\right)=\left[\begin{array}{cccc}
1 & 0 & 0 & 0 \\
0 & 1 & 0 & 0 \\
0 & 0 & 1 & d_{i} \\
0 & 0 & 0 & 1
\end{array}\right] \\
\operatorname{Trans}\left(a_{i}, 0,0\right)=\left[\begin{array}{cccc}
1 & 0 & 0 & a_{i} \\
0 & 1 & 0 & 0 \\
0 & 0 & 1 & 0 \\
0 & 0 & 0 & 1
\end{array}\right] \\
\operatorname{Rot}\left(x, \alpha_{i}\right)=\left[\begin{array}{ccccc}
1 & 0 & 0 & 0 \\
0 & c \alpha_{i} & -s \alpha_{i} & 0 \\
0 & s \alpha_{i} & c \alpha_{i} & 0 \\
0 & 0 & 0 & 1
\end{array}\right]
\end{gathered}
$$

Therefore,

$$
{ }_{i}^{i-1} T=\left[\begin{array}{cccc}
c \theta_{i} & -s \theta_{i} c \alpha_{i} & s \theta_{i} s \alpha_{i} & a_{i} c \theta_{i} \\
s \theta_{i} & c \theta_{i} c \alpha_{i} & -c \theta_{i} s \alpha_{i} & a_{i} s \theta_{i} \\
0 & s \alpha_{i} & c \alpha_{i} & d_{i} \\
0 & 0 & 0 & 1
\end{array}\right]
$$

where $s^{*}$ and $c^{*}$ denotes $\sin ^{*}$ and $\cos ^{*}$.

According to the D-H method, the transformation of links of coordinate system $\{6\}$ relative to $\{0\}$ :
${ }_{6}^{0} T=\left[\begin{array}{cccc}m_{11} & n_{12} & l_{13} & p_{x} \\ m_{21} & n_{22} & l_{23} & p_{y} \\ m_{31} & n_{32} & l_{33} & p_{z} \\ 0 & 0 & 0 & 1\end{array}\right]=\left[\begin{array}{cccc}\vec{m} & \vec{n} & \vec{l} & \vec{p} \\ 0 & 0 & 0 & 1\end{array}\right]$

where $\vec{m}=\left[\begin{array}{lll}m_{11} & m_{21} & m_{31}\end{array}\right]^{T}, \vec{n}=\left[\begin{array}{lll}n_{12} & n_{22} & n_{32}\end{array}\right]^{T}$, $\vec{l}=\left[\begin{array}{lll}l_{13} & l_{23} & l_{33}\end{array}\right]^{T}, \vec{p}=\left[\begin{array}{lll}p_{x} & p_{y} & p_{z}\end{array}\right]^{T}$.

Equation (7) is the forward kinematics model of this 6 -DOF robotic manipulator, where the vectors $(\vec{m}, \vec{n}, \vec{l})$ represent the direction vectors of the three axes $\left(x_{6}, y_{6}, z_{6}\right)$ of the coordinate system $\{6\}$ in the coordinate system $\{0\}$. The vector $\vec{p}$ represents the position of the origin of coordinates $(H)$ of the coordinate system $\{6\}$ in the coordinate system $\{0\}$. We can figure out the position coordinates of the hand claw relative to the base when we substitute the parameters of links and joints into these equations.

\section{DYNAMIC MODEL}

Considering a robotic manipulator of $\mathrm{n}$-joint, its dynamic performance can be described by a second order nonlinear differential equation [6]:

$$
D(q) \ddot{q}+C(q, \dot{q}) \dot{q}+G(q)+\omega=\tau
$$

where $q \in R^{n}$ is the angular displacement of the joint, $D(q) \in R^{n \times n} \quad$ is the positive definite inertia matrix, $C(q, \dot{q}) \in R^{n}$ is the centrifugal force and Coriolis force, $G(q) \in R^{n}$ is gravity, $\tau \in R^{n}$ is control torque, $\omega \in R^{n}$ is the bounded external interference ( if $d>0,\|\omega\|<d$ ).

The kinetic characteristics of the robot system are as follows:

Property 1 [10]-[11]: $D(q)-2 C(q, \dot{q})$ is a skew symmetric matrix;

Property 2 [12]: is a symmetrical positive definite matrix, $\forall \mathrm{m}_{1}, \mathrm{~m}_{2}>0$, and

$$
\mathrm{m}_{1}\|x\|^{2} \leq x^{T} D(q) x \leq \mathrm{m}_{2}\|x\|^{2}
$$

Property 3 [12]: There is a vector so that $D(q), C(q, \dot{q})$ and $G(q)$ satisfy the following linear relationship:

$$
D(q) \vartheta+C(q, \dot{q}) \rho+G(q)=\Phi(q, \dot{q}, \rho, \vartheta) \theta
$$
where $\Phi(q, \dot{q}, \rho, \vartheta) \in R^{n \times n}$ is a regression matrix, $\theta \in R^{n}$ is a parameter vector.

Lemma 1 [13]: If there exists a continuous and positive definite Lyapunov function $V(t)$ satisfying the following conditions, and it is proved that this system is globally uniformly ultimately bounded (GUUB).

$$
V \leq-\beta V(t)+C^{*}
$$

where $\beta$ and $C^{*}$ are two positive constants.

The tracking error variables $e$ is defined as follows: 


$$
e=q-q_{d}
$$

where $q_{d}$ is desired trajectory.

Let

$$
\begin{gathered}
x=\dot{e}+\lambda e \\
\dot{q}_{r}=\dot{q}_{d}-\lambda e
\end{gathered}
$$

where $\lambda$ is a positive constant. Therefore,

$$
x=\dot{e}+\lambda e=\dot{q}-\dot{q}_{d}+\lambda e=\dot{q}-\dot{q}_{r}
$$

According to Property 3 , let $\vartheta=\ddot{q}_{r}, \rho=\dot{q}_{r}$, therefore,

$$
D(q) \ddot{q}_{r}+C(q, \dot{q}) \dot{q}_{r}+G(q)=\Phi\left(q, \dot{q}, \dot{q}_{r}, \ddot{q}_{r}\right) \theta
$$

Substituting (8) into (16):

$$
D(\ddot{q}-\dot{x})+C(\dot{q}-x)+G=\Phi\left(q, \dot{q}, \dot{q}_{r}, \ddot{q}_{r}\right) \theta
$$

Substituting (15) into (17):

$$
D \dot{x}=\tau-\Phi\left(q, \dot{q}, \dot{q}_{r}, \ddot{q}_{r}\right) \theta-\omega-C x
$$

\section{CONTROL DESIGN}

The control law $\tau$ is given as follows:

$$
\tau=-K_{p} e-K_{v} \dot{e}+\Phi\left(q, \dot{q}, \dot{q}_{r}, \ddot{q}_{r}\right) \hat{\theta}+u
$$

where $K_{p}$ and $K_{v}$ are the control gains, and $k_{p i}, k_{v i},(i$ $=1,2 \ldots n)$ are positive constants. $\hat{\theta}$ is the estimated values of $\theta, u$ is the robust control law.

The robust control law $u$ is given as follows:

$$
u=\left\{\begin{array}{cc}
-\frac{x d}{\|x\|} & \|x\| d>\varepsilon \\
-\frac{x d^{2}}{\varepsilon} & \|x\| d \leq \varepsilon
\end{array}\right.
$$

Designing the following Lyapunov function:

$V(t)=\frac{1}{2} x^{T} D x+\frac{1}{2} e^{T} K_{p} e+\frac{1}{2} \lambda e^{T} K_{v} e+\frac{1}{2} \tilde{\theta}^{T} \Gamma \tilde{\theta}$

where $\tilde{\theta}=\theta-\hat{\theta}, \Gamma=\operatorname{diag}\left(\gamma_{1}, \gamma_{2}, \ldots \gamma_{n}\right), \gamma_{i}>0$, $(i$ $=1,2 \ldots n)$. Therefore,

$$
\begin{aligned}
\dot{V}(t) & =x^{T} D \dot{x}+\frac{1}{2} x^{T} \dot{D} x+\dot{e}^{T} K_{p} e+\lambda e^{T} K_{v} \dot{e} \\
& +\tilde{\theta}^{T} \Gamma \dot{\tilde{\theta}}
\end{aligned}
$$

According to (18):

$$
x^{T} D \dot{x}=x^{T}(\tau-\Phi \theta-\omega-C x)
$$

Substituting (19) into (23):

$$
\begin{aligned}
x^{T} D \dot{x} & =x^{T}\left(-K_{p} e-K_{v} \dot{e}+\Phi \hat{\theta}+u-\Phi \theta-\omega-C x\right) \\
& =x^{T}\left(-K_{p} e-K_{v} \dot{e}\right)+x^{T} \Phi \tilde{\theta}+x^{T}(u-\omega) \\
& -x^{T} C x
\end{aligned}
$$

Substituting (24) into (22):

$$
\begin{aligned}
\dot{V}(t)= & x^{T}\left(-K_{p} e-K_{v} \dot{e}\right)+x^{T} \Phi \tilde{\theta}+x^{T}(u-\omega)- \\
& x^{T} C x+\frac{1}{2} x^{T} \dot{D} x+\dot{e}^{T} K_{p} e+\lambda e^{T} K_{v} \dot{e}+\tilde{\theta}^{T} \Gamma \dot{\tilde{\theta}}
\end{aligned}
$$

$$
-x^{T} C x+\frac{1}{2} x^{T} \dot{D} x=\frac{1}{2} x^{T}(\dot{D}-2 C) x=0
$$

and $x^{T}=\dot{e}^{T}+\lambda e^{T}$, therefore,

$$
\begin{aligned}
x^{T}\left(-K_{p} e-K_{v} \dot{e}\right) & =-\dot{e}^{T} K_{p} e-\dot{e}^{T} K_{v} \dot{e}-\lambda e^{T} K_{p} e \\
& -\lambda e^{T} K_{v} \dot{e}
\end{aligned}
$$

Substituting (26) and (27) into (25):

$$
\begin{aligned}
\dot{V}(t) & =-\lambda e^{T} K_{p} e-\dot{e}^{T} K_{v} \dot{e}+x^{T}(u-\omega) \\
& +x^{T} \Phi \tilde{\theta}+\tilde{\theta}^{T} \Gamma \dot{\tilde{\theta}}
\end{aligned}
$$

The adaptive control law is designed as follows:

$$
\dot{\hat{\theta}}=-\Gamma^{-1} \Phi^{T}\left(q, \dot{q}, \dot{q}_{r}, \ddot{q}_{r}\right) x
$$

Substituting (29) into (28):

$$
\dot{V}(t)=-\lambda e^{T} K_{p} e-\dot{e}^{T} K_{v} \dot{e}+x^{T}(u-\omega)
$$

where $x^{T} \Phi \tilde{\theta}=\tilde{\theta}^{T} \Phi^{T} x, \dot{\hat{\theta}}=\dot{\tilde{\theta}}$.

According to Property 2:

$$
\begin{aligned}
& \lambda e^{T} K_{p} e \geq \lambda m_{p 1}\|e\|^{2} \\
& \dot{e}^{T} K_{v} \dot{e} \geq m_{v 1}\|\dot{e}\|^{2}
\end{aligned}
$$

where $m_{p 1}=\min \left\{\mathrm{k}_{p i}\right\}, m_{v 1}=\min \left\{\mathrm{k}_{v i}\right\}$. Therefore,

$\lambda e^{T} K_{p} e+\dot{e}^{T} K_{v} \dot{e} \geq \lambda m_{p 1}\|e\|^{2}+m_{v 1}\|\dot{e}\|^{2} \geq m_{1}\|x\|^{2}$

where $m_{1}=\min \left\{m_{p 1}, m_{v 1}\right\}$.

Substituting (32) into (30):

$$
\dot{V}(t) \leq-m_{1}\|x\|^{2}+x^{T} u-x^{T} \omega
$$

and $-x^{T} \omega \leq\left\|x^{T}\right\| \cdot\|\omega\| \leq\|x\| \cdot d$. Therefore,

$$
\dot{V}(t) \leq-m_{1}\|x\|^{2}+x^{T} u+\|x\| \cdot d
$$

When $\|x\| d>\varepsilon$, and

$$
\dot{V}(t) \leq-m_{1}\|x\|^{2} \leq 0
$$

When $\|x\| d \leq \varepsilon$, and

$$
\dot{V}(t) \leq-m_{1}\|x\|^{2}-\frac{\|x\|^{2} d^{2}}{\varepsilon}+\|x\| d
$$

and when $\|x\| d=\varepsilon / 2$,

$$
\left\{-\frac{\|x\|^{2} d^{2}}{\varepsilon}+\|x\| d\right\}_{\max }=\frac{\varepsilon}{4}
$$

Therefore,

$$
\dot{V}(t) \leq-m_{1}\|x\|^{2}+\frac{\varepsilon}{4}
$$

Integrating (35) and (37):

$$
\dot{V}(t) \leq-m_{1}\|x\|^{2}+\frac{\varepsilon}{4}
$$

According to (21) and Property 2:

$$
\frac{1}{2} m_{1}^{*}\|x\|^{2}+C_{1}^{*} \leq V \leq \frac{1}{2} m_{2}^{*}\|x\|^{2}+C_{2}^{*}
$$

where 

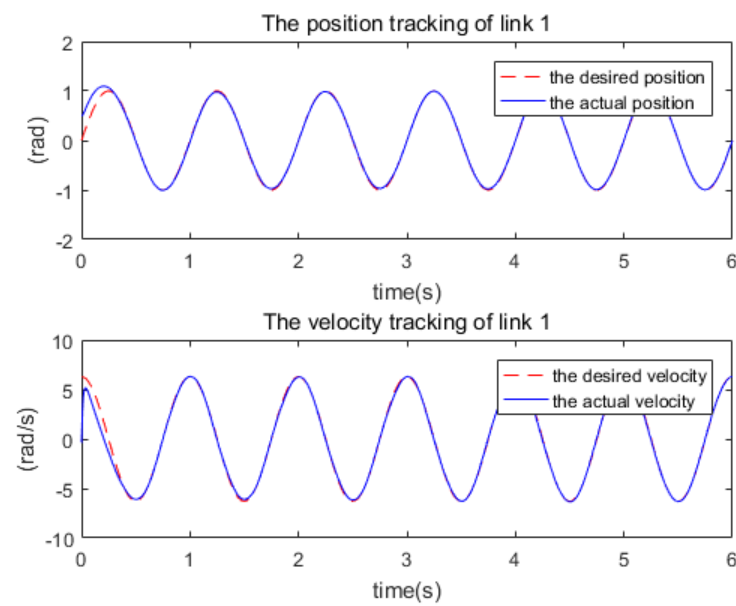

Fig.2. The position tracking and velocity tracking of links.
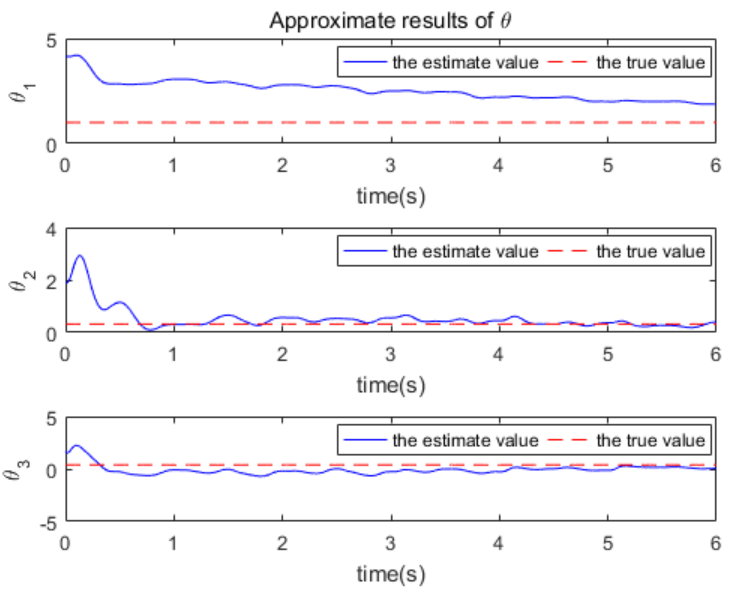

Fig.3. Approximate results of $\theta$ and control input.
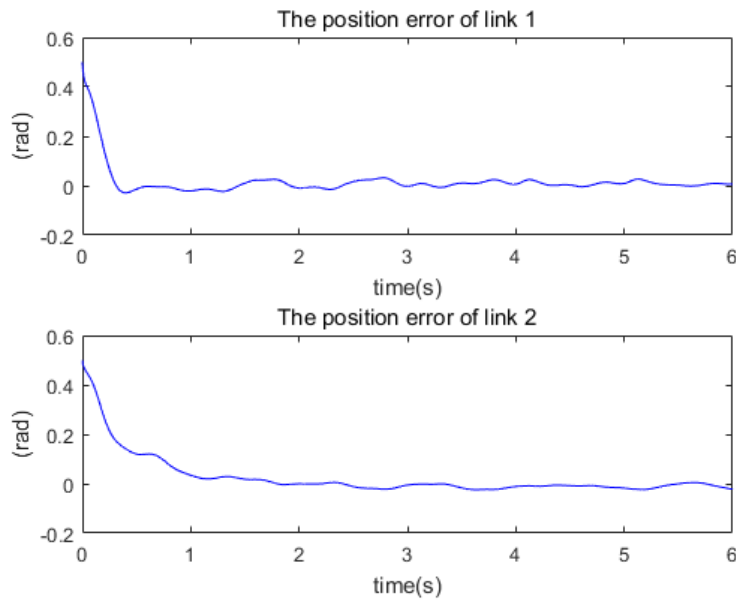

Fig.4. The position tracking error of links.

$$
\begin{gathered}
C_{1}^{*}=\frac{1}{2} m_{p 1}\|e\|^{2}+\frac{1}{2} m_{v 1}\|\dot{e}\|^{2}+\frac{1}{2} \gamma_{\text {min }}\|\tilde{\theta}\|^{2} \\
C_{2}^{*}=\frac{1}{2} M_{p 1}\|e\|^{2}+\frac{1}{2} M_{v 1}\|\dot{e}\|^{2}+\frac{1}{2} \gamma_{\text {max }}\|\tilde{\theta}\|^{2}
\end{gathered}
$$

where

$$
\begin{aligned}
M_{p 1} & =\max \left\{k_{p i}\right\}, M_{v 1}=\max \left\{k_{v i}\right\}, \\
\gamma_{\text {min }} & =\min \left\{\gamma_{i}\right\}, \gamma_{\text {max }}=\max \left\{\gamma_{i}\right\} .
\end{aligned}
$$
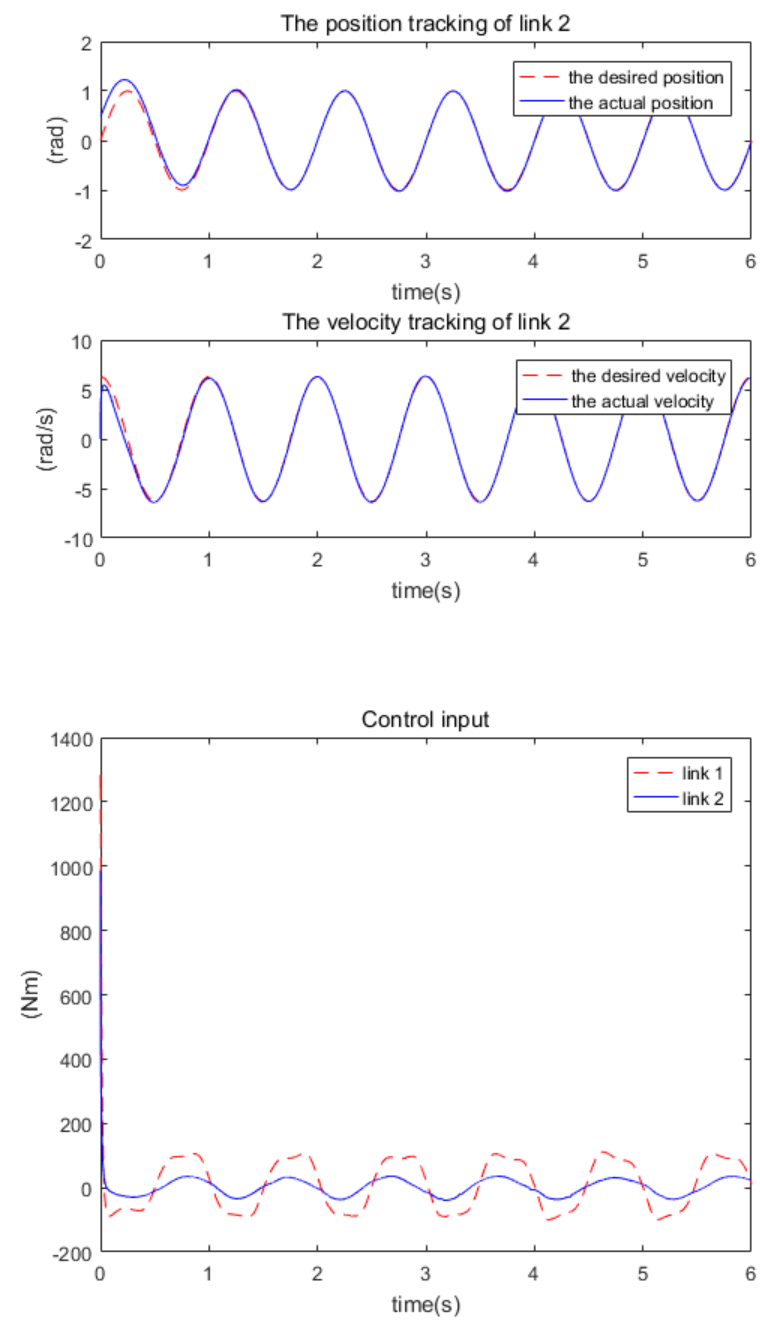

According to (39):

$$
-\|x\|^{2} \leq-\frac{2}{m_{2}^{*}} V+\frac{2}{m_{2}^{*}} C_{2}^{*}
$$

Substituting (40) into (38):

$$
\dot{V}(t) \leq-\frac{2 m_{1}}{m_{2}^{*}} V+\frac{2 m_{1}}{m_{2}^{*}} C_{2}^{*}+\frac{\varepsilon}{4}
$$

According to Lemma 1, the control law (19), (20) and (29) can guarantee that the entire robotic manipulator closed loop system is globally uniformly ultimately bounded.

\section{SimULATION}

In this section, we will verify the effectiveness of the proposed adaptive robust control law for the 2-DOF robotic manipulator. The parameterized model of the 2-DOF robotic manipulator is [10], [14]:

$$
\begin{aligned}
D(q) & =\left[\begin{array}{cc}
\theta_{1}+\theta_{2}+2 \theta_{3} \cos q_{2} & \theta_{2}+\theta_{3} \cos q_{2} \\
\theta_{2}+\theta_{3} \cos q_{2} & \theta_{2}
\end{array}\right] \\
C(q, \dot{q}) & =\left[\begin{array}{cc}
-\theta_{3} \dot{q}_{2} \sin q_{2} & -\theta_{3}\left(\dot{q}_{1}+\dot{q}_{2}\right) \sin q_{2} \\
\theta_{3} \dot{q}_{1} \sin q_{2} & 0
\end{array}\right] \\
G(q) & =\left[\begin{array}{c}
\theta_{1} \gamma \cos q_{2}+\theta_{3} \gamma\left(q_{1}+q_{2}\right) \\
\theta_{3} \gamma\left(q_{1}+q_{2}\right)
\end{array}\right]
\end{aligned}
$$


where $\theta=\left[\begin{array}{lll}\theta_{1} & \theta_{2} & \theta_{3}\end{array}\right]^{T}$ is unknown parameters vector, $\gamma=g / r_{1}, g$ is the gravity acceleration, $g=9.8 \mathrm{~m} / \mathrm{s}^{2}$. $r_{1}, r_{2}$ are the length of links. $m_{1}, m_{2}$ are the mass of links.

The desired position trajectories are as follows:

$$
q_{d 1}=q_{d 2}=\sin (2 \pi t)
$$

The initial states of the system are as follows:

$$
q_{1}(0)=0.5, \dot{q}_{1}(0)=0, q_{2}(0)=0.5, \dot{q}_{2}(0)=0
$$

The external disturbances are as follows:

$$
\omega_{1}=3 \dot{q}_{1} \sin t, \omega_{2}=3 \dot{q}_{2} \cos t
$$

The parameters of the robotic manipulators are as follows:

$$
r_{1}=1.0 \mathrm{~m}, r_{2}=0.8 \mathrm{~m}, m_{1}=0.5 \mathrm{~kg}, m_{2}=0.5 \mathrm{~kg}
$$

The true value of unknown parameters are as follows:

$$
\theta=\left[\begin{array}{lll}
1 & 0.32 & 0.4
\end{array}\right]^{T}
$$

The control parameters are respectively:

$$
\begin{gathered}
K_{p}=\left[\begin{array}{cc}
150 & 0 \\
0 & 150
\end{array}\right], K_{v}=\left[\begin{array}{cc}
150 & 0 \\
0 & 150
\end{array}\right] \\
\lambda=\left[\begin{array}{ll}
5 & 0 \\
0 & 5
\end{array}\right], \Gamma=\left[\begin{array}{ccc}
5 & 0 & 0 \\
0 & 5 & 0 \\
0 & 0 & 5
\end{array}\right] \\
d=3, \varepsilon=0.01
\end{gathered}
$$

Fig. 2 shows the position tracking and the velocity tracking of the robotic manipulator respectively, Fig. 3 and Fig.4 show the approximate results of the unknown parameters, control input and the position tracking error respectively. From the figures, it can be seen that the link 1 can track the desired position around $0.5 \mathrm{~s}$, that is, the position error of link 1 converges to zero. The link 2 can also obtain the same tracking effect around 1s. Because velocity is the derivative of the displacement, the proposed controller ensures that the robotic manipulators track the desired velocity. There is a better effect that the estimate value of $\theta_{2}$ and $\theta_{3}$ converge to the true value compared to $\theta_{1}$.

\section{CONCLUSION}

In this paper, we use D-H method to construct the transformation matrix between each joint of the robotic manipulator, and deduced the positive kinematics model of the robotic manipulator. For the trajectory tracking of robotic manipulators with uncertain external disturbances, an adaptive robust control strategy is proposed. The stability of the closed loop system has been proved by Lyapunov function. Simulation results have been proved that this method is able to guarantee the robotic manipulators track the desired trajectory successfully, where the system error is converging to zero.

\section{REFERENCES}

[1] H. Hu, and G. R. Liu, "On-line adaptive robust neural network tracking control for robot manipulators," Control Theory and Applications, vol. 26, no. 3, pp. 337-341, 2009.

[2] R. Kumar, S. Srivastava, and J. R. P. Gupta, "Online modeling and adaptive control of robotic manipulators using Gaussian radial basis function networks," Neural Computing and Applications, vol. 30, no. 1, pp. 223-239, 2018.
[3] Y. Mo, "Six degree of freedom series improved adaptive robust control algorithm for robot," Journal of Chinese Agricultural Mechanization, vol. 37, no. 5, pp. 232-235+274, 2016.

[4] L. G. Jiang and J. Z. Zhang, "An adaptive control approach for manipulator," Ship Science and Technology, vol. 36, no. 6, pp. 129-133, 2014.

[5] X. J. Mu, "Research on Adaptive Neural Sliding Mode Control of Manipulator Robots," Control Engineering of China, vol. 22, no. 4, pp. 780-784, 2015.

[6] A. T. M. Amin, A. H. A. Rahim and C. Y. Low, "Adaptive controller algorithm for 2-DOF humanoid robot arm," Procedia Technology, no. 15, pp. 765-774, 2014.

[7] Y. L. Xiong, Fundamentals of robotics, "Wuhan: Huazhong University of Science and Technology press," 1996.

[8] X. Zhang, Z. L. Zheng, and Y. Qi, "Parameter Identification and Calibration of D-H Model for 6-DOF Serial Robots," Robot, vol. 38, no. 3, pp. 360-370, 2016.

[9] R. X. Li, F. X. Li, J. M. Yang, and J. Y. Zhou, "Workspace Analysis of Serial Robot Manipulator Based on D-H Method," MACHINE TOOL and HYDRAULICS, vol. 43, no. 21, pp. 70-73+152, 2015.

[10] J. K. Liu, Design and MATLAB simulation of robot control system, "Beijing: Tsinghua university press," 2008.

[11] Y. Li, S. S. Ge, and C. Yang, "Learning impedance control for physical robot-environment interaction," Int. J. Control, vol. 85, no. 2, pp. 182-193, 2012.

[12] X. H. Jiao, Y. F. Li, and et al. "A Robust Adaptive Control Method for Robot," Robotics and Applications, no. 3, pp. 40-43, 2002.

[13] Y. Dai, "The New class of robot robust tracking control strategy," Robot, vol. 20, no. 2, pp. 111-115, 1998.

[14] W. He, Y. T. Dong, and C. Y. Sun, "Adaptive Neural Impedance Control of a Robotic Manipulator with Input Saturation," IEEE TRANSACTIONS ON SYSTEMS, MAN, AND CYBERNETICS: SYSTEMS, vol. 46, no. 3, pp. 334-344, 2016.

[15] J. B. Wen, "Kinematics and dynamics analysis of 6-DOF manipulator and robust trajectory tracking control for uncertain rigid robot," M.S. Thesis, 2002.

[16] S. Singh, and E. Singla, "Modelling and simulation of basic robotic configurations using D-H parameters based adaptive modules," Department of Mechanical Engineering, pp. 513-515, 2016.

[17] S. Singh, A. Singla, and et al, "Kinematic modelling of a five-DOFs spatial manipulator used in robot-assisted surgery," Perspectives in Science, pp. 550-553, 2016.

Bin Ren is currently an associate professor in Department of Mechanical Engineering, Shanghai University. She was as a Hong Kong Scholar at Department of Architectural and Civil Engineering, City University of Hong Kong, Hong Kong, during 2014 to 2016. Her research interests include product digital design, virtual prototype simulation and hybrid control computer.

Yao Wang is currently a graduate student in Department of Mechanical Engineering, Shanghai University, China. His research interest is robotic manipulator and automation.

Li-lan Liu is with Department of Mechanical Engineering, Shanghai Key Laboratory of Intelligent Manufacturing and Robotics, Shanghai University, China.

Xiao-wei Tu is a professor in Department of Mechanical Engineering, Shanghai University, China.

Rogelio Lozano is a professor in Universit de Technologie de Compiegne, CNRS UMR 7253 Heudiasyc, France. 